\title{
Predictors of prostate bed recurrence on magnetic resonance imaging in patients with rising prostate-specific antigen after radical prostatectomy
}

Nicola J. Nasser ${ }^{1}$; Victoria Chernyak ${ }^{2}$; Viswanathan Shankar ${ }^{3}$; Madhur Garg ${ }^{1}$; William Bodner ${ }^{1}$; Shalom Kalnicki ${ }^{1}$; Jonathan Klein ${ }^{1}$

${ }^{1}$ Department of Radiation Oncology, Montefiore Medical Center and Albert Einstein College of Medicine, Bronx, NY, United States; ${ }^{2}$ Departemtn of Radiology, Montefiore Medical Center and Albert Einstein College of Medicine, Bronx, NY, United States; ${ }^{3}$ Department of Epidemiology \& Population Health, Montefiore Medical Center and Albert Einstein College of Medicine, Bronx, NY, United States

Cite as: Can Urol Assoc J 2020 July 17; Epub ahead of print. http://dx.doi.org/10.5489/cuaj.6463

Published online July 17, 2020

$* * *$

\section{Abstract}

Introduction: Radical prostatectomy $(\mathrm{RP})$ is a standard treatment modality for localized prostate cancer. Biochemical failure after RP is usually evaluated with whole body imaging to exclude distant metastatic disease, and pelvic magnetic resonance imaging (MRI) to detect local recurrence in the prostatectomy bed. The goal of this study is to correlate disease characteristics and demographic data in patients with rising prostate-specific antigen (PSA) after RP to determine association with MRI-detected cancer recurrence.

Methods: Sixty-four patients who underwent pelvic MRI for rising PSA after RP and had complete clinical and pathological data available were included. Using Chi-squared testing, we analyzed PSA levels, pathological disease characteristics (prostate cancer risk group, Gleason score, extracapsular extension, positive surgical margin, seminal vesicle involvement, perineural invasion, lymphovascular invasion, and PSA level before MRI), time from surgery to biochemical failure, and patient demographic characteristics as potential predictors of MRdetected local recurrence.

Results: Definite MRI-detected local recurrence was observed in 17/64 patients (27\%). Eleven (17\%) patients had a suspicious lesion with the differential of scarring, retained seminal vesicle, or recurrent cancer. Thirty-six (56\%) patients had no evidence of tumor in the prostate bed or pelvis on MRI. Patient race was associated with likelihood of detecting a prostate nodule on MRI $(p=0.04)$, with African-American patients having $82 \%$ lower odds of MRI-detected tumor 
recurrence compared with White patients $(\mathrm{p}=0.045)$. No other tumor or patient characteristic was significantly associated with MRI-detected recurrence.

Conclusions: African-American patients with biochemical failure after RP are less likely to have MRI-detectable recurrence in the prostate bed compared with White patients.

\section{Introduction}

Prostate cancer is the most prevalent non-cutaneous malignancy among men ${ }^{1}$. Primary treatment options for localized prostate cancer include radical prostatectomy (RP) ${ }^{2}$ or radiation therapy (RT), which may involve external beam $\mathrm{RT}^{3}$ or brachytherapy ${ }^{4,5}$.

Prostate Specific Antigen (PSA) is a human tissue kallikrein expressed in normal and malignant prostate tissues, which becomes undetectable after successful RP ${ }^{6}$. Elevated PSA in blood samples from undetectable levels after RP, termed biochemical failure, suggests recurrence of prostate cancer. Standard workup of a rising PSA after RP includes pelvic imaging, most commonly MRI, to detect local recurrence within the surgical bed and pelvic lymph nodes, and whole-body imaging to evaluate for distant metastatic bone disease. Whole-body imaging techniques include technetium-99m-MDP bone scan and positron emission tomography (PET) scan using prostate-specific membrane antigen (PSMA), C-11 choline, or F-18 fluciclovine ${ }^{7,8}$.

Patients with biochemical failure and no evidence of distant metastatic disease are usually treated with salvage RT to the prostate bed with curative intent. Because RT targeted to the prostate bed does not eradicate distant metastatic disease ${ }^{9}$, a visible nodule in the prostate bed suggests localized recurrence and, thus, potentially increased chance of cure with salvage RT. Previous data suggest salvage RT is associated with a three-fold increase in prostate cancerspecific survival relative to patients who received no salvage treatment ${ }^{10}$. More than $60 \%$ of patients who are treated before the PSA level rises above $0.5 \mathrm{ng} / \mathrm{mL}$ will achieve an undetectable PSA level after salvage RT ${ }^{8,11}$. Initiating RT when PSA is low (e.g. $<0.5 \mathrm{ng} / \mathrm{mL}$ ) has also been shown to improve overall survival ${ }^{12}$.

Prostate bed RT doses of 64-72 Gy are commonly recommended ${ }^{13,14}$, since a multiinstitutional observational study demonstrated that a salvage radiation dose of $\geq 66.0$ Gy was associated with reduced incidence of biochemical failure, but not of distant metastasis ${ }^{15}$. Biochemical control rates following salvage RT increase with higher RT dose and decrease with higher pre-treatment PSA ${ }^{16}$. Clinical practice guidelines ${ }^{14}$ also suggest treating prostate bed nodules that are visible on imaging at the time of recurrence with additional RT, either via simultaneous integrated boost or sequential boost techniques, although no randomized studies have been published demonstrating that boost RT improves control or survival rates ${ }^{17}$. 
Information on predictors of MRI-detected local recurrence may be helpful to guide treatment decisions for patients with biochemical failure after RP. In this study, we investigated factors predictive of MRI-detectable prostate bed nodules among patients with rising PSA after RP.

\section{Methods}

\section{Patient population}

The Montefiore-Einstein Institutional Review Board (IRB) approved this retrospective, Health Insurance Portability and Accountability Act (HIPPA)-compliant analysis, and the need for informed consent was waived. All patients with biochemical failure who underwent MRI of the pelvis after having had a previous RP at a single tertiary care center between January 2013 and December 2017 were included in this study. Patients were identified using a commercial decision support tool (Looking Glass Clinical Analytics, Streamline Health), and the following data were collected for all eligible patients:

1. Demographics: Birthdate, race, ethnicity, and date of RP.

2. PSA blood levels: PSA values before and after RP, nadir PSA, time to nadir PSA, and PSA near the MRI scan.

3. Pathology reports (from RP): Gleason Score, resection margin involvement, seminal vesicle (SV) involvement, extracapsular extension (ECE), lymphovascular invasion (LVI) and perineural invasion (PNI).

\section{MRI scans}

Clinical MRI reports were reviewed, including the provided clinical indication. Exams are read by faculty radiologists ( $\mathrm{n}=5$ during the study period) with fellowship training and expertise in abdominal imaging, each with 3 or more years of post-fellowship experience in MRI of the prostate. Each exam was read and approved by one of the five radiologists.

At our institution, prostate MRIs are performed on 1.5T or 3T scanners (Ingenia, Philips Healthcare, Best, Netherlands) using multiparametric techniques. During the study period, the examinations were performed in accordance to technical specifications proposed by the Prostate Imaging-Reporting and Data System (PI-RADS) manual. Multiparametric MRI included small field-of-view axial, coronal, sagittal T2-weighted images, axial diffusion-weighted sequences, dynamic contrast-enhanced sequences, and large field-of-view axial pre- and post-contrast T1weighted images through the entire pelvis. Recurrence was identified as asymmetric area with differential signal intensity, detected on any of the obtained sequences. The examinations are performed with a surface coil, but without an endorectal coil.

Patients with suspected prostate cancer recurrence were included in the study. Included patients had not undergone RT to the prostate or pelvis prior to their MRI to investigate prostate cancer recurrence. Based on the radiology report, patients were classified as having definite 
recurrent disease, no recurrent disease, or equivocal for recurrent disease. Patients with definite recurrent disease were those where MRI definitively demonstrated a recurrent tumor, patients with no recurrent disease were those with no detectable abnormality to suggest macroscopic recurrence, and patients with findings equivocal for recurrent disease were those with a reported asymmetric abnormality on MRI, with the differential of scarring, retained SV or recurrent cancer.

Patients were stratified into risk groups (low, favorable intermediate, unfavorable intermediate, high, and very high) according to the National Comprehensive Cancer Network $(\mathrm{NCCN})$ guidelines based on preoperative PSA, Gleason score, ECE, and SV involvement ${ }^{14}$.

\section{Statistical analysis}

Patients' clinical and demographic data were summarized numerically using descriptive statistics. Continuous scale variables that are normally distributed were summarized using mean and standard deviation, while median and range were used for variables that were not normally distributed. Three sets of sensitivity analyses were performed by categorizing those patients with equivocal MRI findings as (i) definite recurrence, (ii) no recurrence, and (iii) excluding them from the analysis entirely. Comparison of clinical characteristics between patients MRI confirmed recurrence and those with negative results were tested using student t-test; non-normal variables were tested using Wilcoxon rank-sum test. Categorical variables were tested either using chi-square test or Fisher's exact test as appropriate. A logistic regression model was fitted to examine the association between recurrence and covariates.

\section{Results}

Initially, 76 patients were identified by CLG software. Eleven patients were excluded from analysis: seven patients did not have previous prostate cancer, one patient had an intact prostate visible on MRI, one patient received hormonal therapy before radical prostatectomy, and for two patients the pathology was not available. One patient was found to have low-risk disease and was excluded from analysis. Figure 1 shows a Consolidated Standards of Reporting Trials (CONSORT) diagram describing patient inclusion in this study. Sixty-four patients had pelvic MRI after RP for evaluation of prostate cancer recurrence and had complete data available. Clinical and demographic characteristics are presented in Table 1. The average age of patients at the time of RP was 59.6 years (range 40-76). Median PSA prior to surgery was $9.8 \mathrm{ng} / \mathrm{ml}$ (range: 0.46 - 114). Two-thirds of the patient had high or very high-risk disease (per NCCN criteria) at the time of RP.

\section{MRI recurrence results}

Definite local recurrence of prostate cancer on MRI was found in 17 (27\%) of 64 patients (Table 2). Representative images of these tumor recurrences are presented in Figure 2. Thirty-six (56\%) had no evidence of recurrence on MRI. Eleven (17\%) had MRI report with findings equivocal 
for recurrence (Table 2).

For the primary analysis, we considered patients with equivocal MRI findings as definite recurrence (Table 3, Sample 1). In this analysis, MRI-confirmed local disease was observed more often among white patients compared with African American patients ( $70 \%$ vs 30\%). Compared with white race, the estimated odds of MR-detected local disease were $82 \%$ less among African American patients ( $\mathrm{OR}=0.18,95 \% \mathrm{CI}: 0.04-0.96, \mathrm{p}=0.045)$, and $77 \%$ less among Hispanic patients (OR=0.23, 95\% CI: 0.05-1.10, $\mathrm{p}=0.065)$.

Two secondary sensitivity analyses were performed. In the first sensitivity analysis, patients with equivocal MRI results were considered as negative (Table 3, Sample 2). MRIconfirmed local disease was observed more often among white patients compared with African American patients (50\% vs $15 \%$ ). Compared with white patients, the estimated odds of MRdetected local disease were $82 \%$ less among African American patients ( $\mathrm{OR}=0.176,95 \% \mathrm{CI}$ : $0.03-1.01, \mathrm{p}=0.0513)$, and $76 \%$ less among Hispanic patients $(\mathrm{OR}=0.238,95 \% \mathrm{CI}: 0.05-1.15, \mathrm{p}$ $=0.0745)$.

In the second sensitivity analysis, patients with equivocal MRI results were not included (Table 3, Sample 3). MRI-confirmed local disease was observed more often among white patients compared with African American patients (63\% vs 18\%). Compared with white patients, the estimated odds of MR-detected local disease were $87 \%$ less among African American patients $(\mathrm{OR}=0.129,95 \% \mathrm{CI}: 0.02-0.86, \mathrm{p}=0.0342)$, and $82 \%$ less among Hispanic patients $(\mathrm{OR}=0.176,95 \% \mathrm{CI}: 0.03-1.15, \mathrm{p}=0.0513)$.

None of the other examined characteristics including prostate cancer risk group, Gleason score, ECE, positive surgical margin (defined as tumor present at the inked margin), SV involvement, PNI, LVI, and PSA level before MRI were significantly associated with MRIdetected recurrence in any of our three analyses. Results of all three analyses are shown in Table 3 .

\section{Discussion}

In this retrospective cohort of patients with rising PSA after radical prostatectomy, 27\% of patients were found to have MRI-detectable prostate bed recurrence, while $17 \%$ had a radiologically-apparent nodule equivocal for disease recurrence. Logistic regression modelling found that patients' self-declared race and ethnicity was the only significant predictor of MRIdetected local recurrence, with African-American patients having $82 \%$ lower odds of MRIdetected tumor recurrence compared with white patients. A trend toward lower MRI-detected recurrence rates among Hispanic patients was also seen, although did not reach statistical significance. Other factors which are predictive of outcomes in prostate cancer such as PSA level, Gleason score, surgical margin status, and prostate cancer risk group did not predict MRIdetectable local recurrence in our cohort. This finding may be due to the relatively small number of patients, the retrospective nature of our study or due to different tumor biology presenting 
among different patient populations (e.g. higher propensity for African American patients to develop aggressive prostate cancer and recur distantly).

Known risk factors for biochemical failure after RP include PSA level before surgery, Gleason score, seminal vesicle involvement, extracapsular extension, positive surgical margins, and perineural involvement ${ }^{18-22}$. Anscher et al. ${ }^{23}$ found that positive surgical margins, poorly differentiated histology, and elevated acid phosphatase were significant predictors of local recurrence after prostatectomy. Stephenson et al. ${ }^{9}$ found that positive surgical margins, long PSA doubling time ( $>10$ months), and PSA level $<2 \mathrm{ng} / \mathrm{mL}$ at the time of salvage treatment were positive predictive factors for survival after salvage RT following BF. Because RT acts only locally in the prostate bed, in the case of salvage RT, improved salvage rates among patients with these risk factors suggests higher likelihood of local recurrence (compared to metastatic spread) in patients with these factors.

Previous studies have investigated recurrence patterns and risk factors for local compared with distant recurrence after BF. Giovacchini et al. ${ }^{24}$ used C-11 choline PET to investigate patterns of recurrence in patients with biochemical failure after RP. Prostate bed recurrence was observed in 55/368 patients (15\%) compared with 107 (29\%) patients with lymph node recurrence and $46(13 \%)$ with recurrence in the bones. PET/CT or PET/MRI with C-11 choline could provide more data with regard to reccurences in the prostate bed by correlating anatomic and metabolic data. A retrospective study that investigated the clinical impact of $\mathrm{C}-11$ choline $\mathrm{PET} / \mathrm{CT}$ on treatment management decisions in patients with recurrent prostate cancer after radical therapy, showed that it led to an overall treatment change in $46.7 \%$ of patients, with a major clinical change implemented in $18 \%$ of patients (such as changing the decision of palliative androgen deprivation therapy, before $\mathrm{C}-11$ choline PET/CT to salvage therapy after $\mathrm{PET} / \mathrm{CT})^{25}$.

Hernandez et al. ${ }^{26}$ reported on a cohort of 70 patients from Spain imaged with multiparametric MRI. Mean PSA before MRI was $0.38 \mathrm{ng} / \mathrm{ml}$. Local recurrence was identified in 33 patients $(47 \%)$. No factors predicted for detectable prostate bed nodules including PSA, pathologic cancer stage, lymph node positivity, Gleason score, bladder neck involvement, LVI, location of surgical margin or whether pelvic lymph node dissection was performed ${ }^{26}$. Our results, from a cohort with similar characteristics to this study, also suggested a similar lack of predictive ability of pathologic and PSA markers for predicting MRI-detectable local recurrence.

Endorectal MRI (eMRI) has been suggested as an improved method for detecting local recurrence after RP, with reported sensitivity and specificity ranging from $84-97 \%$ and $74-100 \%$, respectively ${ }^{27-30}$. Using a variety of sequences obtained with $3 \mathrm{~T}$ eMRI including T2-weighted imaging, dynamic contrast-enhanced (DCE), and diffusion-weighted imaging (DWI), Kitajima et al. ${ }^{31}$ studied a cohort of 80 patients with evidence of recurrence based on biopsy, PSA reduction after salvage treatment (e.g. RT), or MRI finding of a growing suspicious pelvic lesion. In this cohort of patients with high suspicion of local recurrence, eMRI detected recurrence in 57/80 
patients $(71 \%)$. The PSA value at the time of MRI in this cohort was higher than in our cohort (mean $1.17 \mathrm{ng} / \mathrm{mL}$ and median $0.43 \mathrm{ng} / \mathrm{mL}$ versus $0.8 \mathrm{ng} / \mathrm{mL}$ and $0.3 \mathrm{ng} / \mathrm{mL}$ in our cohort, respectively). Liauw et al. ${ }^{32}$ studied 88 men with detectable PSA after RP without clinically palpable disease who agreed to undergo eMRI. The mean PSA value before MRI was $0.3 \mathrm{ng} / \mathrm{mL}$. Using eMRI, 24\% of patients had detectable local recurrence. Univariate analysis suggested that the highest post-operative PSA value was a significant predictor of local recurrence, with 0.3 $\mathrm{ng} / \mathrm{mL}$ acting as the most predictive cut-off. Age, Gleason score, T stage, $\mathrm{N}$ stage, positive surgical margins, and MRI magnet strength (1.5 vs $3 \mathrm{~T}$ ) were not predictive. Race/ethnicity was not studied.

In our cohort, eMRI was not used. Mean PSA prior to MRI was $0.3 \mathrm{ng} / \mathrm{mL}$ in our cohort, similar to that measured in Hernandez et al ${ }^{26}$ (without eMRI) and Liauw et al ${ }^{32}$ (with eMRI). Definite evidence of recurrence was seen in $27 \%$ of patients in our cohort, similar to the $24 \%$ detected by Liauw et al. ${ }^{32}$ but lower than the $47 \%$ reported by Hernandez et al. ${ }^{26}$. The difference between the rates reported by Hernandez et al. and our study could be that $17 \%$ of our patients had equivocal disease recurrence which may have been reported as positive in that cohort.

The relationship between race and prostate cancer has been previously studied. One previous report found that African Americans have higher rates of recurrence following RP ${ }^{33}$. Another recent study found that, after adjustment for nonbiological differences (notably access to care and standardized treatment), African-American patients did not have different stage-forstage prostate cancer specific mortality ${ }^{34}$. Steele et al. ${ }^{35}$ reported that among 1,527,602 prostate cancers diagnosed between 2001 and 2009 in the USA, the proportion of localized cases increased from $73 \%$ to $77 \%$ in black males and from $77 \%$ to $79 \%$ in white males, compared to the years 1990-1994 ${ }^{36}$, and net survival exceeded $99 \%$ for localized stage in both racial groups.

The differences in prostate cancer rates by race, ethnicity, and geography may be explained by variation in access to screening and treatment as well as variation in the underlying biology of prostate carcinogenesis. Some groups have a genomic propensity to develop biologically aggressive disease, with men of African descent having higher prostate cancer incidence and poorer prognosis due to more aggressive disease at diagnosis ${ }^{37}$. These factors could account for the differences in the patterns of recurrence with relation to race reported in our study, since African-American patients may be more likely to harbor micrometastatic disease at initial diagnosis, leading to higher rates of distant failure rather than local failure when disease recurs.

Our results suggest that African-American patients with biochemical failure after RP may be less likely to have MRI-detectable recurrence in the prostate bed compared with white patients. Analysis of subsequent survival data can help us understand if patients with an imagingdetected prostate bed nodule have a higher chance of cure than those without a detectable nodule. Lack of a radiologically-detected macroscopic disease after biochemical failure suggests 
microscopic disease recurrence, which may be associated with either local (and salvageable) recurrence or distant (non-salvageable) recurrence.

Serum PSA levels should be undetectable after successful prostate cancer cure via radical prostatectomy. Any detectable PSA levels with currently available commercial essays should prompt investigatory imaging including pelvic MRI. Ultrasensitive PSA assays could be beneficial in detecting persistent PSA after radical prostatectomy ${ }^{38,39}$.

We expect that patterns of local and systemic failure after prostate brachytherapy or external beam radiation therapy for prostate cancer would be similar to those observed in the cohort presented here. Proving this, however, would be more challenging as prostate biopsies (likely multiple) would be needed in addition to MRI to accurately detect and characterize local recurrence in the setting of an intact prostate.

The main limitations of our study are its retrospective nature and a relatively small cohort size, although our sample was similar in number to previous similar publications. We used the MRI clinical report to discriminate between patients with or without local recurrence, rather than reviewing the scans again. This method was used to reflect and be applicable to real-world clinical practice patterns. Larger prospective cohorts and longer follow-up will help further refine the factors predictive local recurrence and the significance of these predictions in personalizing treatments and improving outcomes for all prostate cancer patients.

\section{Conclusions}

African-American patients with biochemical failure after RP are less likely to have MRIdetectable recurrence in the prostate bed compared with white patients.

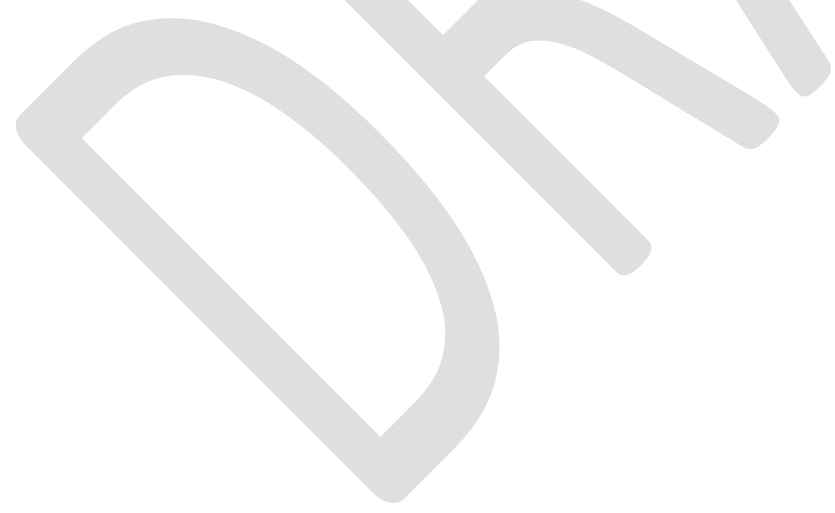




\section{References}

1. Siegel RL, Miller KD, Jemal A. Cancer statistics, 2019. CA: a cancer journal for clinicians 2019;69:7-34.

2. D'Amico AV, Whittington R, Malkowicz S, et al. Biochemical outcome after radical prostatectomy, external beam radiation therapy, or interstitial radiation therapy for clinically localized prostate cancer. JAMA 1998;280:969-74.

3. Thor M, Jackson A, Zelefsky MJ, et al. Inter-institutional analysis demonstrates the importance of lower than previously anticipated dose regions to prevent late rectal bleeding following prostate radiotherapy. Radiotherapy and oncology : journal of the European Society for Therapeutic Radiology and Oncology 2018;127:88-95.

4. Nasser NJ, Wang Y, Borg J, Saibishkumar EP. Sector analysis of dosimetry of prostate cancer patients treated with low-dose-rate brachytherapy. Brachytherapy 2014;13:36974.

5. Nasser NJ, Sappiatzer J, Wang Y, Borg J, Saibishkumar EP. Dosimetric evaluation of clinical target volume in the postimplant analysis of low-dose-rate brachytherapy for prostate cancer. Brachytherapy 2015;14:189-96.

6. Nasser NJ, Thoms J, Soosaipillai A, et al. Human tissue Kallikreins: Blood levels and response to radiotherapy in intermediate risk prostate cancer. Radiotherapy and oncology : journal of the European Society for Therapeutic Radiology and Oncology 2017;124:427-32.

7. Froemming AT, Verma S, Eberhardt SC, et al. ACR Appropriateness Criteria((R)) Posttreatment Follow-up Prostate Cancer. Journal of the American College of Radiology : JACR 2018;15:S132-s49.

8. EAU-ESTRO-ESUR-SIOG guidelines on prostate cancer. EAU - ESTRO - ESUR SIOG, 2018. (Accessed 8/9/2019, 2019, at https://uroweb.org/wp-content/uploads/EAUESUR-ESTRO-SIOG-Guidelines-on-Prostate-Cancer-large-text-V2.pdf.)

9. Stephenson AJ, Scardino PT, Kattan MW, et al. Predicting the outcome of salvage radiation therapy for recurrent prostate cancer after radical prostatectomy. Journal of clinical oncology : official journal of the American Society of Clinical Oncology 2007;25:2035-41.

10. Trock BJ, Han M, Freedland SJ, et al. Prostate cancer-specific survival following salvage radiotherapy vs observation in men with biochemical recurrence after radical prostatectomy. Jama 2008;299:2760-9.

11. Wiegel T, Lohm G, Bottke D, et al. Achieving an Undetectable PSA After Radiotherapy for Biochemical Progression After Radical Prostatectomy Is an Independent Predictor of Biochemical Outcome\&\#x2014;Results of a Retrospective Study. International Journal of Radiation Oncology • Biology• Physics 2009;73:1009-16.

12. Stish BJ, Pisansky TM, Harmsen WS, et al. Improved Metastasis-Free and Survival Outcomes With Early Salvage Radiotherapy in Men With Detectable Prostate-Specific Antigen After Prostatectomy for Prostate Cancer. Journal of clinical oncology : official journal of the American Society of Clinical Oncology 2016;34:3864-71. 
13. Rathbun JT, Schroeder TM, Eberhardt SC. High postprostatectomy prostate-specific antigen level prior to salvage radiation therapy is not always a bad sign. Reviews in urology 2017;19:190-4.

14. Prostate Cancer. National Comprehensive Cancer Network, 2019. (Accessed 07/14/2019, 2019, at https://www.nccn.org/professionals/physician_gls/pdf/prostate.pdf.)

15. Pisansky TM, Agrawal S, Hamstra DA, et al. Salvage Radiation Therapy Dose Response for Biochemical Failure of Prostate Cancer After Prostatectomy-A MultiInstitutional Observational Study. International journal of radiation oncology, biology, physics 2016;96:1046-53.

16. Ohri N, Dicker AP, Trabulsi EJ, Showalter TN. Can early implementation of salvage radiotherapy for prostate cancer improve the therapeutic ratio? A systematic review and regression meta-analysis with radiobiological modelling. Eur J Cancer 2012;48:837-44.

17. Beck M, Wust P, Barelkowski T, et al. Risk adapted dose-intensified postoperative radiation therapy in prostate cancer patients using a simultaneous integrated boost technique applied with helical Tomotherapy. Radiation oncology (London, England) 2017;12:125.

18. Bolla M, van Poppel H, Collette L, et al. Postoperative radiotherapy after radical prostatectomy: a randomised controlled trial (EORTC trial 22911). Lancet (London, England) 2005;366:572-8.

19. D'Amico AV, Wu Y, Chen MH, Nash M, Renshaw AA, Richie JP. Perineural invasion as a predictor of biochemical outcome following radical prostatectomy for select men with clinically localized prostate cancer. The Journal of urology 2001;165:126-9.

20. Kupelian PA, Katcher J, Levin HS, Klein EA. Stage T1-2 prostate cancer: a multivariate analysis of factors affecting biochemical and clinical failures after radical prostatectomy. International journal of radiation oncology, biology, physics 1997;37:1043-52.

21. Swanson GP, Riggs M, Hermans M. Pathologic findings at radical prostatectomy: risk factors for failure and death. Urologic oncology 2007;25:110-4.

22. Bolla M, van Poppel H, Tombal B, et al. Postoperative radiotherapy after radical prostatectomy for high-risk prostate cancer: long-term results of a randomised controlled trial (EORTC trial 22911). Lancet (London, England) 2012;380:2018-27.

23. Anscher MS, Prosnitz LR. Multivariate analysis of factors predicting local relapse after radical prostatectomy--possible indications for postoperative radiotherapy. International journal of radiation oncology, biology, physics 1991;21:941-7.

24. Giovacchini G, Picchio M, Coradeschi E, et al. Predictive factors of [(11)C]choline $\mathrm{PET} / \mathrm{CT}$ in patients with biochemical failure after radical prostatectomy. European journal of nuclear medicine and molecular imaging 2010;37:301-9.

25. Ceci F, Herrmann K, Castellucci P, et al. Impact of 11C-choline PET/CT on clinical decision making in recurrent prostate cancer: results from a retrospective two-centre trial. European journal of nuclear medicine and molecular imaging 2014;41:2222-31.

26. Hernandez D, Salas D, Gimenez D, et al. Pelvic MRI findings in relapsed prostate cancer after radical prostatectomy. Radiation oncology (London, England) 2015;10:262. 
27. Kitajima K, Murphy RC, Nathan MA, et al. Detection of recurrent prostate cancer after radical prostatectomy: comparison of $11 \mathrm{C}$-choline PET/CT with pelvic multiparametric MR imaging with endorectal coil. Journal of nuclear medicine : official publication, Society of Nuclear Medicine 2014;55:223-32.

28. Cirillo S, Petracchini M, Scotti L, et al. Endorectal magnetic resonance imaging at 1.5 Tesla to assess local recurrence following radical prostatectomy using T2-weighted and contrast-enhanced imaging. European radiology 2009;19:761-9.

29. Sella T, Schwartz LH, Swindle PW, et al. Suspected local recurrence after radical prostatectomy: endorectal coil MR imaging. Radiology 2004;231:379-85.

30. Casciani E, Polettini E, Carmenini E, et al. Endorectal and dynamic contrast-enhanced MRI for detection of local recurrence after radical prostatectomy. AJR American journal of roentgenology 2008;190:1187-92.

31. Kitajima K, Hartman RP, Froemming AT, Hagen CE, Takahashi N, Kawashima A. Detection of Local Recurrence of Prostate Cancer After Radical Prostatectomy Using Endorectal Coil MRI at 3 T: Addition of DWI and Dynamic Contrast Enhancement to T2-Weighted MRI. AJR American journal of roentgenology 2015;205:807-16.

32. Liauw SL, Pitroda SP, Eggener SE, et al. Evaluation of the prostate bed for local recurrence after radical prostatectomy using endorectal magnetic resonance imaging. International journal of radiation oncology, biology, physics 2013;85:378-84.

33. Moul Judd W, Douglas Thomas H, McCarthy William F, McLeod David G. Black Race is an Adverse Prognostic Factor for Prostate Cancer Recurrence Following Radical Prostatectomy in an Equal Access Health Care Setting. Journal of Urology 1996; $155: 1667-73$.

34. Dess RT, Hartman HE, Mahal BA, et al. Association of Black Race With Prostate Cancer-Specific and Other-Cause Mortality. JAMA oncology 2019.

35. Steele CB, Li J, Huang B, Weir HK. Prostate cancer survival in the United States by race and stage (2001-2009): Findings from the CONCORD-2 study. Cancer 2017;123 Suppl 24:5160-77.

36. Coleman MP, Quaresma M, Berrino F, et al. Cancer survival in five continents: a worldwide population-based study (CONCORD). The Lancet Oncology 2008;9:730-56.

37. Rebbeck TR. Prostate Cancer Genetics: Variation by Race, Ethnicity, and Geography. Seminars in radiation oncology 2017;27:3-10.

38. Kinoshita H, Kamoto T, Nishiyama H, Nakamura E, Matsuda T, Ogawa O. Prostate specific antigen nadir determined using ultra-sensitive prostate specific antigen as a predictor of biochemical progression after radical prostatectomy in Japanese males. International journal of urology : official journal of the Japanese Urological Association 2007;14:930-4; discussion 4.

39. Oesterling JE, Tekchandani AH, Martin SK, et al. The periurethral glands do not significantly influence the serum prostate specific antigen concentration. The Journal of urology 1996;155:1658-60. 


\section{Figures and Tables}

Fig. 1. Consolidated Standards of Reporting Trials (CONSORT) flowchart. ADT: androgen deprivation therapy.

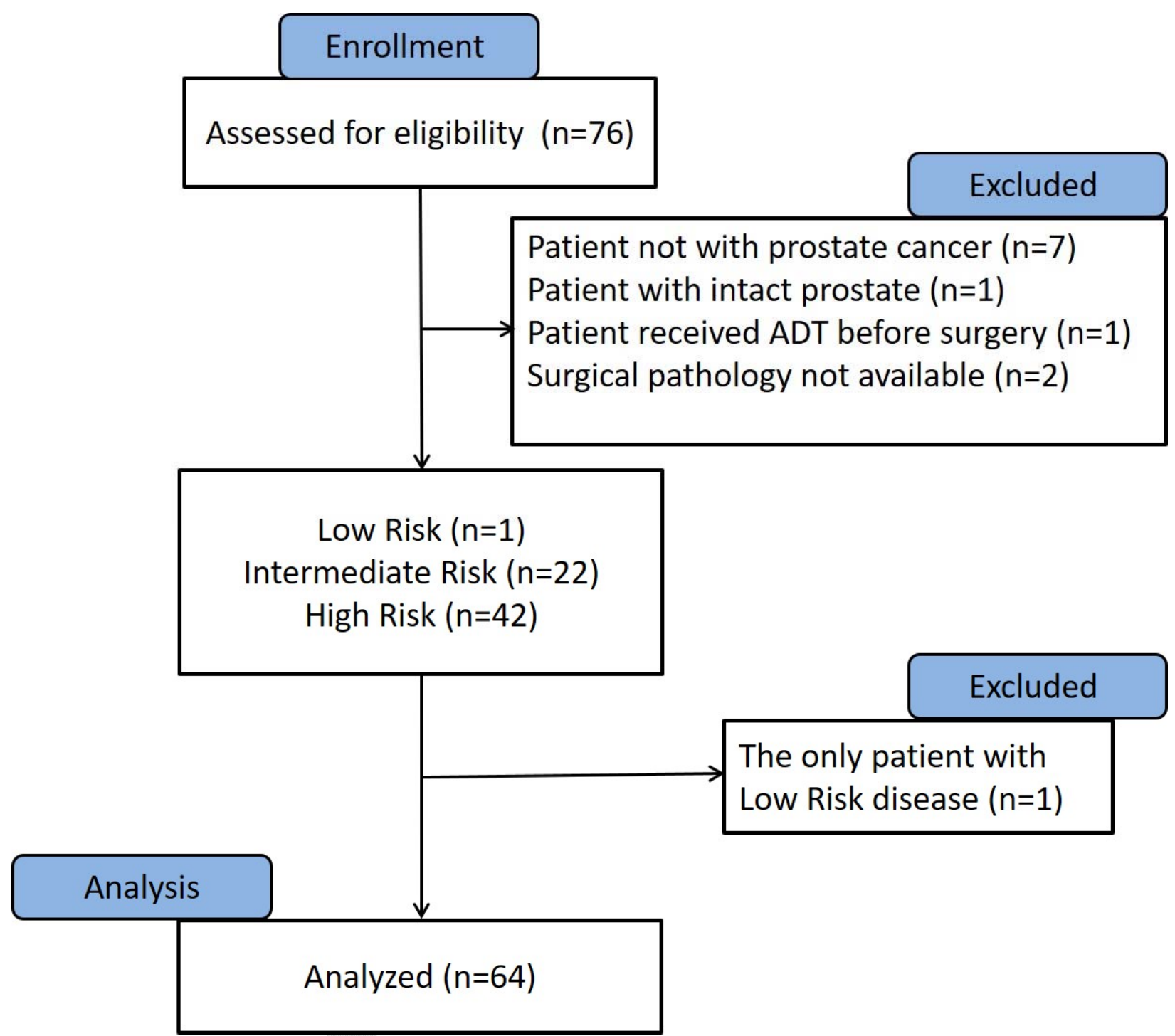


Fig. 2. Prostate bed nodule compatible with local recurrence of prostate cancer (white arrows) was found in 17 patients (27\%). Representative images from 16 patients are presented.
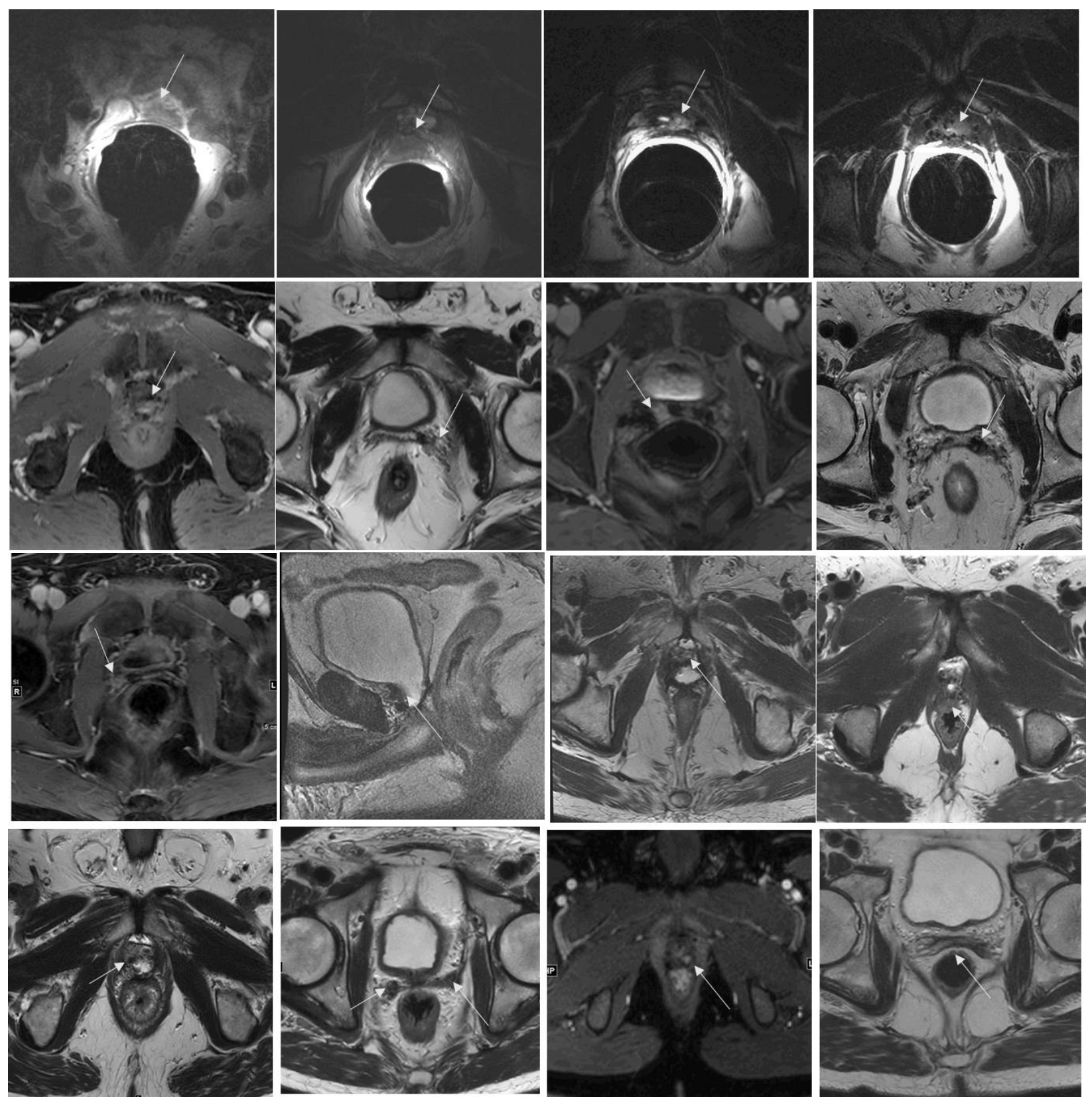


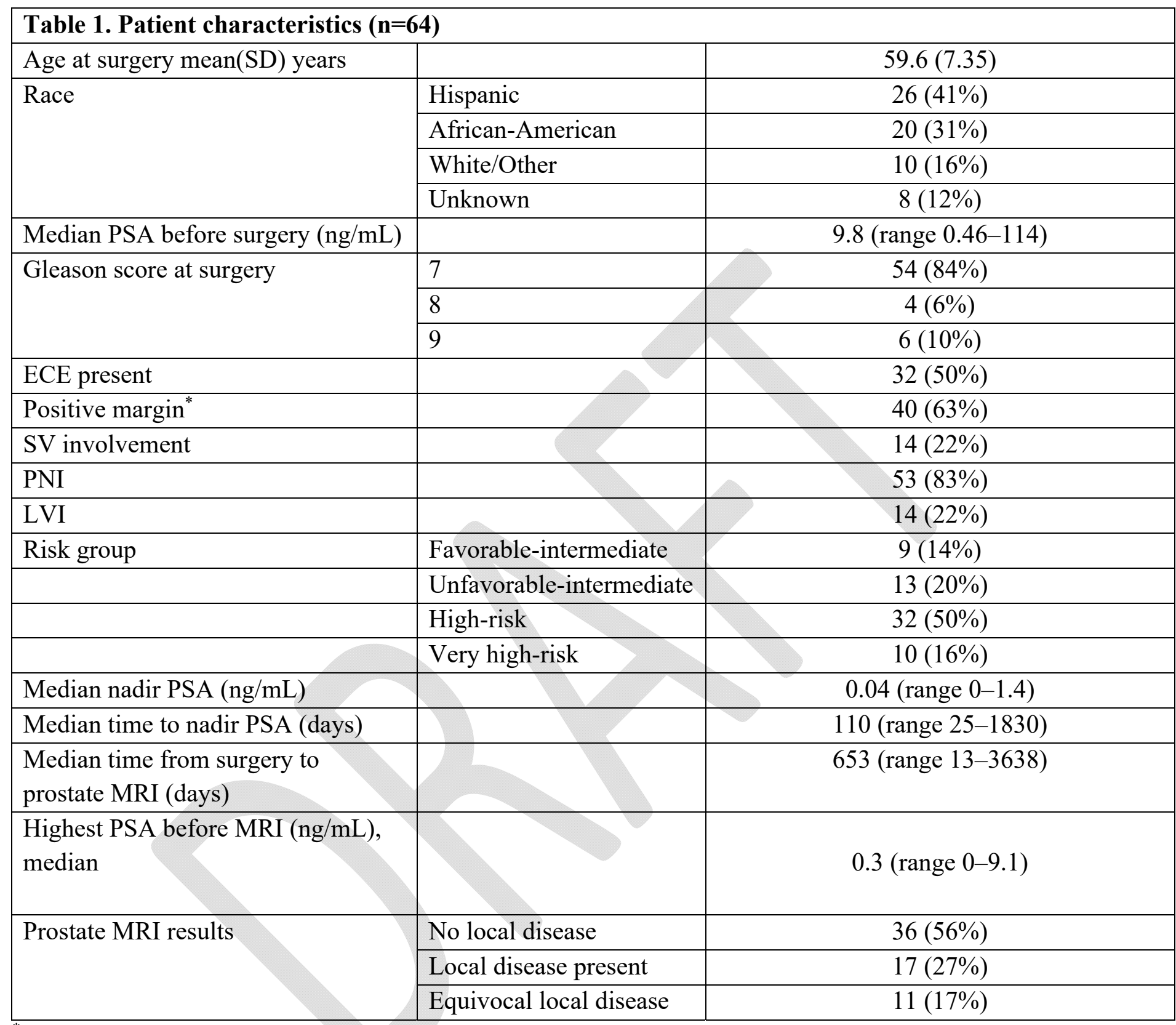

${ }^{*}$ Defined as tumor at the inked surgical margin. ECE: extracapsular extension; LVI: lymphovascular invasion; MRI: magnetic resonance imaging; PNI: perineural invasion; PSA: prostate-specific antigen; SV: seminal vesicles. 


\begin{tabular}{|l|c|c|}
\hline Table 2. Location of definitive and equivocal recurrences detected on MRI \\
\hline & $\begin{array}{c}\text { Definite } \\
\text { recurrence, } \mathbf{n}=\mathbf{1 7} \\
\mathbf{( \% )}\end{array}$ & $\begin{array}{c}\text { Equivocal } \\
\text { recurrence, } \mathbf{n = 1 1} \\
\mathbf{( \% )}\end{array}$ \\
\hline Prostatectomy bed & $7(41 \%)$ & $5(45 \%)$ \\
\hline Adjacent to the urinary bladder & $6(35 \%)$ & $1(9 \%)$ \\
\hline Urethral anastomosis & $3(18 \%)$ & $2(18 \%)$ \\
\hline $\begin{array}{l}\text { Superior aspect of urogenital } \\
\text { diaphragm }\end{array}$ & $1(6 \%)$ & 0 \\
\hline Perivesical space & 0 & $3(27 \%)$ \\
\hline
\end{tabular}

MRI: magnetic resonace imaging.

\begin{tabular}{|c|c|c|c|c|c|c|}
\hline \multirow[b]{2}{*}{ Covariates } & \multicolumn{2}{|c|}{ Sample $1^{*}$} & \multicolumn{2}{|c|}{ Sample $2^{* *}$} & \multicolumn{2}{|c|}{ Sample $3^{* * *}$} \\
\hline & $\begin{array}{l}\text { Odds ratio } \\
\text { (95\% CI) }\end{array}$ & $\mathbf{p}$ & $\begin{array}{l}\text { Odds ratio } \\
(95 \% \text { CI })\end{array}$ & $\mathbf{p}$ & $\begin{array}{l}\text { Odds ratio } \\
\text { (95\% CI) }\end{array}$ & $\mathbf{p}$ \\
\hline \multicolumn{7}{|l|}{ Race/ethnicity } \\
\hline African American & $0.18(0.04,0.96)$ & 0.045 & $0.18(0.03,1.01)$ & 0.051 & $0.13(0.02,0.89)$ & 0.034 \\
\hline Hispanic & $0.23(0.05,1.10)$ & 0.065 & $0.24(0.05,1.15)$ & 0.075 & $0.18(0.03,1.01)$ & 0.051 \\
\hline Unknown & $1.28(0.16,10.45)$ & 0.814 & $1.00(0.16,6.42)$ & 1.00 & $1.20(0.13,11.05)$ & 0.872 \\
\hline White/Other & Reference & & Reference & & Reference & \\
\hline \multicolumn{7}{|l|}{ High vs. low } \\
\hline $\mathrm{H} / \mathrm{VH}$ & $2.14(0.73,6.32)$ & 0.168 & $1.36(0.41,4.52)$ & 0.616 & $1.71(0.50,5.90)$ & 0.393 \\
\hline FI/UI & Reference & & Reference & & Reference & \\
\hline \multicolumn{7}{|l|}{ Gleason score } \\
\hline 7 & $1.20(0.30,4.74)$ & 0.795 & $0.48(0.12,1.95)$ & 0.302 & $0.65(0.16,2.70)$ & 0.553 \\
\hline$>7$ & Reference & & Reference & & Reference & \\
\hline \multicolumn{7}{|l|}{ ECE present } \\
\hline Yes & $1.82(0.66,4.99)$ & 0.246 & $1.34(0.43,4.20)$ & 0.613 & $1.61(0.49,5.26)$ & 0.433 \\
\hline No & Reference & & Reference & & Reference & \\
\hline \multicolumn{7}{|l|}{ Positive margins } \\
\hline Yes & $1.15(0.41,3.19)$ & 0.795 & $2.41(0.68,8.49)$ & 0.172 & $2.07(0.56,7.63)$ & 0.275 \\
\hline No & Reference & & Reference & & Reference & \\
\hline
\end{tabular}




\begin{tabular}{|c|c|c|c|c|c|c|}
\hline $\begin{array}{c}\text { SV involvement } \\
\text { Yes } \\
\text { No }\end{array}$ & $\begin{array}{c}1.45(0.44,4.78) \\
\text { Reference }\end{array}$ & 0.541 & $\begin{array}{c}0.76(0.18,3.14) \\
\text { Reference }\end{array}$ & 0.699 & $\begin{array}{c}0.96(0.21,4.29) \\
\text { Reference }\end{array}$ & 0.954 \\
\hline $\begin{array}{c}\text { PNI } \\
\text { Yes } \\
\text { No }\end{array}$ & $\begin{array}{c}6.25(0.72,54.39) \\
\text { Reference }\end{array}$ & 0.097 & $\begin{array}{c}2.76(0.31,24.41) \\
\text { Reference }\end{array}$ & 0.360 & $\begin{array}{c}3.75(0.42,33.39) \\
\text { Reference }\end{array}$ & 0.236 \\
\hline $\begin{array}{c}\text { LVI } \\
\text { Yes }\end{array}$ & $\begin{array}{c}0.48(0.13,1.74) \\
\text { Reference }\end{array}$ & 0.262 & $\begin{array}{c}0.77(0.18,3.25) \\
\text { Reference }\end{array}$ & 0.725 & $\begin{array}{c}0.63(0.15,2.69) \\
\text { Reference }\end{array}$ & 0.529 \\
\hline $\begin{array}{c}\text { PSA near MRI } \\
>0.3 \\
\leq 0.3\end{array}$ & $\begin{array}{c}0.52(0.19,1.41) \\
\text { Reference }\end{array}$ & 0.198 & $\begin{array}{c}0.67(0.22,2.06) \\
\text { Reference }\end{array}$ & 0.485 & $\begin{array}{c}0.56(0.17,1.80) \\
\text { Reference }\end{array}$ & 0.331 \\
\hline
\end{tabular}

OR, 95\% CI, and p are provided for each parameter. Sample 1: equivocal MRI results counted as positives; sample 2: equivocal results counted as negatives; sample 3: equivocal results excluded from the analysis. "Equivocal MRI results counted as positives. ** Equivocal results counted as negatives.

${ }^{* * *}$ Equivocal results excluded from the analysis. CI: confidence interval; ECE: extracapsular extension; LVI: lymphovascular invasion; MRI: magnetic resonance imaging; OR: odds ratio; PNI: perineural invasion; PSA: prostate-specific antigen; SV: seminal vesicle. 